University of Nebraska - Lincoln

DigitalCommons@University of Nebraska - Lincoln

\title{
An Epitope from Acanthamoeba castellanii That Cross-React with Proteolipid Protein 139-151-Reactive T Cells Induces Autoimmune Encephalomyelitis in SJL Mice
}

\author{
Chandirasegaran Massilamany \\ University of Nebraska-Lincoln, cmassilamany@unl.edu \\ David Steffan \\ University of Nebraska - Lincoln \\ Jay Reddy \\ University of Nebraska - Lincoln, jayreddy@unl.edu
}

Follow this and additional works at: https://digitalcommons.unl.edu/vbsjayreddy

Part of the Biological Phenomena, Cell Phenomena, and Immunity Commons, Immune System Diseases Commons, Immunopathology Commons, Medical Biochemistry Commons, Medical Molecular Biology Commons, and the Nervous System Diseases Commons

Massilamany, Chandirasegaran; Steffan, David; and Reddy, Jay, "An Epitope from Acanthamoeba castellanii That Cross-React with Proteolipid Protein 139-151-Reactive T Cells Induces Autoimmune Encephalomyelitis in SJL Mice" (2010). Jay Reddy Publications. 17.

https://digitalcommons.unl.edu/vbsjayreddy/17

This Article is brought to you for free and open access by the Veterinary and Biomedical Sciences, Department of at DigitalCommons@University of Nebraska - Lincoln. It has been accepted for inclusion in Jay Reddy Publications by an authorized administrator of DigitalCommons@University of Nebraska - Lincoln. 


\title{
An Epitope from Acanthamoeba castellanii That Cross-React with Proteolipid Protein 139-151-Re- active T Cells Induces Autoimmune Encephalomyelitis in SJL Mice
}

\author{
Chandirasegaran Massilamany, David Steffen, Jay Reddy \\ School of Veterinary Medicine and Biomedical Sciences, University of Nebraska-Lincoln, Lincoln, Nebraska, U.S.A. \\ Corresponding author, Reddy: jayreddy@unl.edu
}

Article history: received October 21, 2009; received in revised form November 10, 2009; accepted November 10, 2009.

Keywords: Experimental autoimmune encephalomyelitis, Multiple sclerosis, Molecular mimicry, Proteolipid protein, Acanthamoeba castellanii, Acanthamoeba granulomatous encephalitis

Abstract

We report here that an epitope (aa, 83-95) derived from Acanthamoeba castellanii (ACA) induces clinical signs of experimental autoimmune encephalomyelitis (EAE) in SJL/J mice reminiscent of the disease induced with myelin proteolipid protein (PLP) 139-151. By using IAs/tetramers, we demonstrate that both ACA 83-95 and PLP 139-151 generate antigen-specific cross-reactive CD4 T cells and the T cells secrete identical patterns of cytokines and induce EAE with a similar severity. These results may provide insights into the pathogenesis of multiple sclerosis and ACA-induced granulomatous encephalitis.

\section{Introduction}

Multiple sclerosis (MS) is a disease of the central nervous system (CNS) characterized by inflammation and infiltration of mononuclear cells and the loss of myelin sheath encapsulating the axons (Sospedra and Martin, 2005). Autoimmune responses to myelin antigens have been implicated in MS pathogenesis and this requires the mediation of autoreactive $T$ cells and $B$ cells, but the mechanisms by which the disease is initiated are unknown (Kerlero de Rosbo et al., 1993; Sospedra and Martin, 2005). Although genetic susceptibility is a major predisposing factor, exposure to environmental microbes such as viruses and bacteria have been suspected in the initiation of autoimmune diseases. Several mechanisms have been postulated to explain this phenomenon. These include the release of self-antigens or cryptic or new antigenic determinants in the target organs leading to the generation of pathogenic CD4 T cells (Miller et al., 1997), bystander activationofautoaggressive $\mathrm{T}$ cells by superantigens (Brocke et al., 1993), and molecular mimicry in which structural homologies between self and foreign antigens facilitate the recognition of self tissues by cross-reactivity (Wucherpfennig and Strominger, 1995).

Molecular mimicry is a natural phenomenon and several examples exist to provide a proof of concept that the microbial mimics can break self-tolerance and induce autoimmune responses in various animal studies, including experimental autoimmune encephalomyelitis (EAE), the disease model for MS in humans (Fujinami et al., 2006; Gautam et al., 1998; Mokhtarian et al., 1999). EAE can be induced by active immunization with emulsions containing myelin antigens in complete Freund's adjuvant (CFA) or by adoptively transferring myelin antigen (Ag)-specific T cells into the susceptible animals (Tuohy et al., 1989; Whitham et al., 1991). Three major myelin antigens or their peptides have been found to induce EAE in rodent or non-rodent species. These are myelin basic protein (MBP) (Zamvil et al., 1986), myelin oligodendrocyte glycoprotein (MOG) (Mendel et al., 1995), and proteolipid protein (PLP) (Tuohy et al., 1989). The microbial mimics capable of inducing EAE have been reported for all the above except for PLP. These include Herpes virus saimiri (Gautam et al., 1998), Hepatitis B virus (Fujinami and Oldstone, 1985), JC virus (Mao et al., 2007), Chlamydia pneumoniae (Conant and Swanborg, 2003), Papilloma virus (MBP mimics) (Ruiz et al., 1999), and Semliki forest virus (MOG mimic) (Mokhtarian et al., 1999).

Our studies involve PLP 139-151-induced EAE in SJL mice, which develop chronic relapsing-remitting paralysis. We sought to identify the disease-producing microbial mimics for PLP 139-151 from pathogens capable of inducing CNS disease and searched for homologous sequences between PLP 139-151 and pathogens in pro- tein databases. This search resulted in the identification of one novel peptide spanning aa 83-95 of rhodanese-related sulfurtransferase in Acanthamoeba castellanii (ACA) and it induces EAE similar to that of PLP 139-151.

\section{Materials and methods}

2.1. Mice

Four to six-week-old female SJL/J (H-2 $)$ mice were obtained from the Jackson Laboratory (Bar Harbor, Maine). The mice were maintained in accordance with the animal protocol guidelines of the University of Nebraska-Lincoln, Lincoln, Nebraska.

\subsection{Peptide synthesis and immunization procedures}

PLP 139-151 (HSLGKWLGHPDKF), ACA 83-95 (YFLLKWLGHPNVS) and neuraminidase (NASE) 101-120 (EALVRQGLAKVAYVYKPNNT) were synthesized on 9-fluorenylmethyloxycarbonyl chemistry (Neopeptide, Cambridge, Massachusetts). All peptides were HPLC-purified (N90\%) and confirmed by mass spectroscopy. To measure recall responses $100 \mu \mathrm{g}$ of each peptide emulsified in CFA was administered subcutaneously in the flank. For disease induction, Mycobacterium tuberculosis (MTB) H37RA extract (Difco Laboratories, Detroit, Michigan) was added as an additional component to a final concentration of $5 \mathrm{mg} / \mathrm{ml}$. Pertussis toxin (List Biological Laboratories, Campbell, California) was administered (100 ng per mouse) intraperitoneally on day 0 and day 2 postimmunization.

\subsection{Identification of microbial peptides that mimic PLP 139-151}

PLP 139-151 is an immunodominant epitope in which the critical residues required for major histocompatibility complex (MHC) class II and T cell receptor (TCR)-binding have been well-characterized (Figure 1). By using PLP 139-151 as a putative antigen, we performed pattern search using the prosite scan of the Bioinformatics Toolkit (http://toolkit.tuebingen.mpg.de/patsearch, Max-Planck Institute for Developmental Biology, Tubingen, Germany). The pattern that we used was XXLXX $\underline{W}$ X $\underline{H} P X X X$ (underlined: TCR-contact residues; bold: MHC-anchor residues; $X$ : non-critical residues).

\subsection{Clinical scoring and histopathology}

Following EAE induction, the mice were monitored for clinical signs of disease and scored as described previously (Tuohy et al., 1989): 0, healthy; 1 , limp tail or hind limb weakness, but not both; 2 , limp tail and hind limb weakness; 3 , partial paralysis of hind limbs; 4, complete paralysis of hind limbs; 5, moribund or dead. Animals were euthanized during recovery or upon termination on day 32 postimmunization. Brain and spinal cords were collected in $10 \%$ 
phosphate buffered formalin and analyzed for histological evidence of the disease (Sobel et al., 1990). After fixation, two brain sections were made one included cerebrum, and hippocampus, the second included cerebellum, and brainstem. In the spinal cord, three sections were made from each segment (cervical, thoracic, lumbar, and sacral). All the tissues were stained by Hematoxylin and Eosin staining. Tissues were blinded to treatment and lesion type characterized and severity scored. Severity scores were obtained by counting inflammatory foci in both meninges and parenchyma for all sites. Inflammation was primarily classified as lymphocytic, suppurative or mixed. For statistical analysis counts were added across all sections of brain and spinal cord for each mouse.

\subsection{Induction of EAE by adoptive transfer}

Short-term $\mathrm{T}$ cell lines were generated from the mice immunized with $100 \mu \mathrm{g}$ of each PLP 139-151 or ACA 83-95 per mouse. Lymph node cells (LNC) obtained from the draining lymph nodes were stimulated with the corresponding peptides $(20 \mu \mathrm{g} / \mathrm{ml})$ and rested for 14 days in interleukin (IL)-2 medium. Antigen-specific T cells were enriched by restimulating with PLP 139-151 or ACA 83-95 (20 $\mu \mathrm{g} / \mathrm{ml}$ ) in the presence of syngeneic Ag-presenting cells for two days and maintained in IL-2 medium for additional two days. Viable lymphoblasts were harvested by Ficoll-Hypaque density gradient centrifugation, and groups of naive SJL mice were injected in the tail vein with $8.5 \times 10^{6}$ cells per mouse (Whitham et al., 1991).

\subsection{Proliferation Assay}

Single cell suspensions were prepared from the draining lymph nodes harvested from groups of mice immunized with PLP 139-151 or ACA 83-95. LNC were stimulated with PLP 139-151, ACA 83-95 and NASE $101-120(0-100 \mu \mathrm{g} / \mathrm{ml})$ at a cell density of 5 to $7.5 \times 10^{6}$ cells $/ \mathrm{ml}$ for two days in RPMI medium supplemented with $10 \%$ fetal calf serum, $1 \mathrm{mM}$ sodium pyruvate, $4 \mathrm{mM}$ L-glutamine, $1 \times$ each of non-essential amino acids and vitamin mixture and $100 \mathrm{U} / \mathrm{ml}$ penicillin-streptomycin (Lonza, Walkersville, Maryland). Cultures were then pulsed with $1 \mu \mathrm{Ci}$ of tritiated ${ }^{3}[\mathrm{H}]$ thymidine per well and $16 \mathrm{~h}$ later, proliferative responses were measured as counts per minute (cpm) using Wallac liquid scintillation counter (Perkin Elmer, Waltham, Massachusetts).

2.7. Derivation of MHC class II/IA $\mathrm{IA}^{\mathrm{s}}$ tetramers and flow cytometric analysis of antigen-specific T cells by tetramer staining

PLP 139-151 and Theiler's murine encephalomyelitis virus (TMEV) 70-86 tetramers were generated as described (Reddy et al., 2003). To derive ACA 83-95 tetramers, IA ${ }^{s}$ construct (gift from Dr. Vijay Kuchroo, Harvard Medical School, Boston, Massachusetts) was modified by excising PLP 139151 sequence using Kpn I and BamH I and replaced with the nt sequence encoding ACA 83-95 (5'-TATTTTCTTCTTAAATGGCTTGGTCATCCTAATGTTTCT-3'). The soluble IA molecules were expressed in Baculovirus expression system by in-

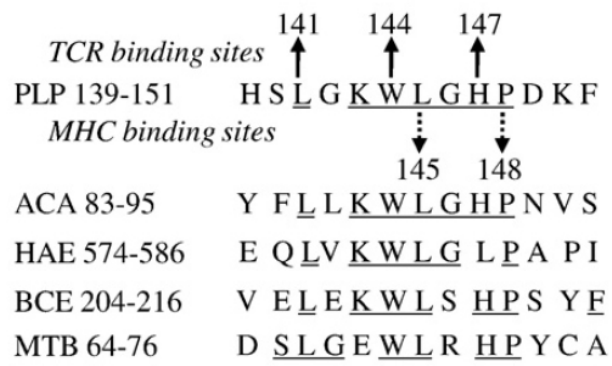

Figure 1. Comparison of microbial peptide sequences with PLP 139-151. The peptide sequences of Acanthamoeba castellanii (ACA), Haemophilus influenzae (HAE), Bacillus cereus (BCE), and Mycobacterium tuberculosis (MTB) are compared with proteolipid protein (PLP) 139-151. Homologous sequences are underlined. fecting SF9 insect cells using a high-titer virus supernatant and the tetramers were derived as described previously (Reddy et al., 2003). To determine the frequency of Ag-specific T cells, LNC obtained from the mice immunized with PLP 139-151 or ACA 83-95 were restimulated with the corresponding peptides $(20 \mu \mathrm{g} / \mathrm{ml})$ for four to six days. Viable lymphoblasts were harvested by Ficoll-Hypaque density gradient centrifugation and incubated with phycoerythrin (PE) conjugated IAs tetramers $(30 \mu \mathrm{g} / \mathrm{ml})$ at room temperature (RT) for $3 \mathrm{~h}$ followed by staining with anti-CD4 and 7-amino-actinomycin-D (7-AAD) (Eugene, Oregon) (Reddy et al., 2003, 2004). The frequency of tetramer ${ }^{+}\left(\right.$tet $\left.^{+}\right)$cells was then enumerated by four-color flow cytometric analysis (FACS caliber, BD Pharmingen, San Diego, California) and Flow Jo software (Tree star, Ashland, Oregon) in a live (7-AAD-) $\mathrm{CD}^{+}$population. To determine the $\mathrm{Ag}$ specificity of cross-reactive T cell responses induced with PLP 139-151 and ACA 83-95, double tetramer staining was performed using allophycocyanin (APC)-conjugated PLP 139-151-and ACA 83-95-PE tetramers.

2.8. Intracellular cytokine staining

LNC from PLP 139-151 or ACA 83-95-immunized groups were restimulated with the respective peptides and maintained in medium containing IL-2. Viable lymphoblasts were harvested on day four by Ficoll-Hypaque density gradient centrifugation and stimulated for $4.5 \mathrm{~h}$ with Phorbol 12-myristate 13-acetate (PMA) $(10 \mathrm{ng} / \mathrm{ml})$ and Ionomycin $(150 \mathrm{ng} / \mathrm{ml})$ in the presence of $2 \mathrm{mM}$ monensin (Golgi stop, BD Pharmingen). After staining with anti-CD4 and 7-AAD, cells were fixed, permeabilized and stained with cytokine antibodies or isotype controls (eBioscience, San Diego, California). The frequency of cytokine-secreting cells was then determined in the live (7-AAD) $\mathrm{CD}^{+}$subset by flow cytometry (FACS Scan, BD Pharmingen) and analyzed by Flow Jo software (Tree star) (Reddy et al., 2004). The clones of cytokine antibodies used were: IL-2 (JES6-5H4), IL-4 (11B11), IL-10 (JES5-16E3), interferon (INF)- $\gamma$ (XMG1.2), tumor necrosis factor (TNF)- (MP6XT22), IL-17A (eBio 17B7), IL-17F (eBio 18F10) (all from eBioscience) and IL-22 (140301) (R\&D Systems, Minneapolis, Minnesota).

\subsection{Cytokine ELISA}

Supernatants harvested from the above cultures on day two were analyzed for cytokines by ELISA (Nicholson et al., 1995; Reddy et al., $2004)$. The clones of the capture and detection antibody $(\mathrm{Ab})$ pairs used were: IL-2 (JES6-1A12/JES6-5H4), IL-4 (11B11/BVD6-24G2), IL-10 (JES5-16E3/JES5-2A5), IL-17A (eBio17CK15A5/eBio17B7), INF-ץ (AN-18/R4-6A2), and TNF-a (TN3-19.12/rabbit polyclonal) (eBioscience).

\subsection{Statistics}

Student's $t$-test was used to determine differences in T cell proliferative responses, tetramer staining, and cytokine secretion between groups. Comparisons of histological disease between groups for inflammatory lesions in the brain and spinal cords were analyzed by two-sided Wilcoxon rank sum test (Mann-Whitney $U$ test). $P \leq 0.05$ values were considered significant.

\section{Results}

3.1. An epitope derived from A. castellanii induces autoimmune encephalomyelitis

Protein database searches resulted in the identification of seventeen sequences derived from organisms that are known to be associated with CNS infections in humans with a percent homology of 38.4 to 53.8 with PLP 139-151 (Table 1). To determine whether or not the microbial mimics that bear homologous sequences with PLP 139151 induce CNS autoimmunity, we chose ACA 83-95, Bacillus cereus (BCE) 204-216 and MTB 64-76 based on two criteria: (a) Peptides should possess a maximum sequence homology with PLP 139-151 and (b) the homologous sequences should include all of the critical TCR and MHC Contact residues (Table 1 and Figure 1). Among the three peptides tested, only ACA 83-95 induced the clinical signs of 
EAE by active immunization. There were no clinical signs of EAE in the mice immunized with BCE and MTB peptides, nor was there consistent evidence of inflammation on histological evaluation of CNS tissues (data not shown).

Comparison of disease severity and patterns of clinical EAE induced with PLP 139-151 and ACA 83-95 revealed a few noteworthy observations (Figure 2a and Table 2). First, the onset of EAE induction was similar for both PLP 139-151 and ACA 83-95 peptides ( day 11 ) and among the clinically affected mice, the mean maximum neurological scores were marginally higher for PLP 139-151 when compared with mice immunized with ACA 83-95 (3.39 vs. 2.20) (Table 2). Second, EAE occurred in all of the mice immunized with PLP 139-151 and $72.7 \%(16 / 22)$ of the mice immunized with ACA 83-95 showed signs of EAE clinically and/or histologically. Of these, $45 \%$ $(10 / 22)$ of mice showed both clinical and histological EAE (Figure 2aand Table 2) and the remaining six mice (27.3\%) while showing no clinical signs of EAE had histological evidence of disease. Third, consistent with the clinical EAE, histologically, the severity of the disease was significantly milder in mice immunized with ACA 83-95 than in mice immunized with PLP 139-151 as revealed by fewer foci of inflammation in the brain and spinal cords ( $p=1.6624$ E-23) (Table 2 and Figure 3i). When evident, regardless of clinical EAE or not, infiltrates in both PLP 139-151-and ACA 83-95-immunized groups primarilycomprisedperivascular cuffing of lymphocytes with few histiocytes and plasma cells of the brain and spinal cords as the disease severity increased. Mixed as reported previously (Sobel et al., 1990). Inflammation was limited to infiltrates made of both neutrophils and MNC were present in most meninges in mice with mild EAE, but it was extended into the parenchyma severe lesions.

3.2. ACA 83-95-and PLP 139-151-reactive T cells induce EAE with a similar severity

Short-term T cell lines were obtained from SJL mice immunized with PLP 139-151 and ACA 83-95 and viable lymphoblasts were administered into groups of naïve SJL mice. The T cells reacting with each individual peptide induced clinical EAE of similar course, severity and histology beginning at day five posttransfer (Figures $2 \mathrm{~b}$ and 3ii and Table 2). Infiltrations comprised of MNC and neutrophils were present in both groups of mice.

3.3.ACA83-95inducesTcellresponsesthatcross-reactwithPLP139-151 To measure recall responses, we used LNC obtained from mice immunized with ACA 83-95 or PLP 139-151. As shown in Figure 4, both PLP 139-151 and ACA 83-95 induced comparable T cell responses in a dose-dependent manner and the response was specific, because NASE 101-120 (control peptide) failed to stimulate T cells from either

Table 1

Microbial peptides that mimic CNS myelin PLP 139-151.

Source

PLP 139-151

Acanthamoeba castellanii

Bacillus anthracis str. Ames

Bacillus cereus G9241

Bacillus cereus NVH0597-99

Chaetomium globosum CBS 148.51

Mycobacterium tuberculosis CDC1551 DSLGEWLRHPYCA

Mycobacterium avium 104

Mycobacterium avium sub sp. paratuberculosis

Mycobacterium tuberculosis H37Rv

Nostoc punctiforme PCC73102

Nostoc sp. PCC 7120

Borna disease virus

Trichinella spiralis

Nematostella vectensis

$\begin{array}{lc}\text { Sequence } & \text { Homology }(\%) \\ \text { HSLGKWLGHPDKF } & \\ \text { YFLLKWLGHPNVS } & 53.8 \\ \text { VELEKWLRHPSYF } & 53.8 \\ \text { VELEKWLSHPSYF } & 53.8 \\ \text { VKLEKWLSHPSYF } & 53.8 \\ \text { HHLPSWLGHPTRV } & 53.8 \\ \text { DLLGEWLRHPYCA } & 53.8 \\ \text { ESLGEWLR } \underline{\text { HPYCA }} & 53.8 \\ & \\ \text { ESLGEWLRHPYCA } & 53.8 \\ \text { DSLGEWLRHPYCA } & 53.8 \\ \text { TDLEVWLGHPGKV } & 53.8 \\ \text { TDLEVWLGHPGKV } & 53.8 \\ \text { QFLRSWLNHPDII } & 46.1 \\ \text { PPLSTWLGHPNHS } & 46.1 \\ \text { CGLCAWLIHPCV } & 38.4 \\ \text { CCLCVWLIHPCNV } & 38.4 \\ \text { CCLCVWLIHPCIV } & 38.4 \\ \text { CFLCVWLIHPCIV } & 38.4 \\ \text { ed. }\end{array}$

Homologous residues are underlined.

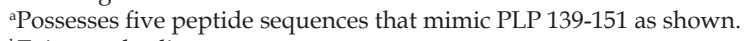
${ }^{b}$ Exists as duplicate sequence. group. Importantly, LNC obtained from PLP 139-151-immunized group also reacted to ACA 83-95 albeit at a lower level as compared with the native peptide (PLP 139-151) (Figure 4a). Likewise, LNC from ACA 83-95-immunized mice also reacted to PLP 139-151, but the response was low (Figure 4b). Marginally, the magnitude of T cell response to PLP 139-151 in ACA 83-95-immunized mice was lower than the reactivity to ACA 83-95 in PLP 139-151-immunized mice.

3.4. Cross-reactive $\mathrm{T}$ cell responses induced with ACA 83-95 and PLP 139-151 are antigen-specific

To determine if the expansion of $\mathrm{T}$ cells that occurred in ACA 8395-immunized mice react specifically with PLP 139-151, we used IAs tetramers for PLP 139-151. Flow cytometric analysis of tet+ cells indicated that about $2 \%$ of the CD4 cells in PLP 139-151- and $1 \%$ in ACA 83-95-immunized mice stained with PLP 139-151 tetramers implying that ACA 83-95 generates PLP 139-151-specific T cells (Figure $5 \mathrm{a})$. To verify the variations, if any, in the generation of precursor frequency of PLP 139-151-specific T cells in mice immunized with PLP 139-151 and ACA 83-95, we developed tetramers for ACA 83-95. Consistent with the proliferative responses, PLP 139-151 tet+ cells in PLP139-151-immunized mice and ACA 83-95 tet+ cells in ACA 83-95 immunized mice were evident as predicted (Figure $5 b$ ). The stain-

\section{(a) Active immunization}

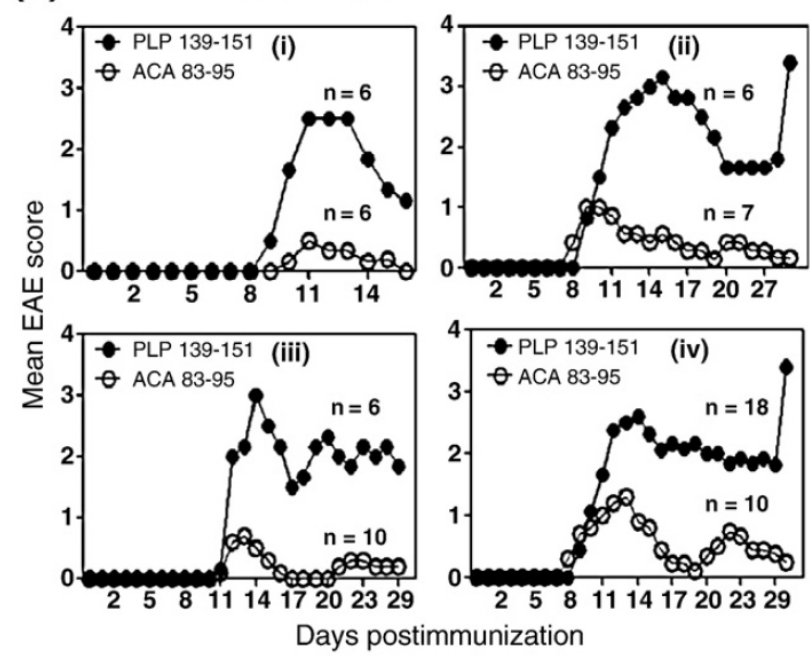

\section{(b) Adoptive transfer}

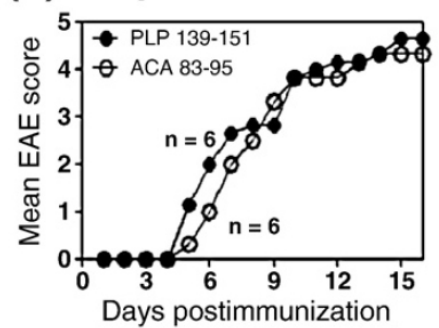

Figure 2. Induction of EAE by PLP 139-151 and ACA 83-95. (a) Induction of EAE by active immunization. SJL mice were immunized with $100 \mu \mathrm{g}$ each of PLP 139-151 or ACA 83-95 in CFA and pertussis toxin was administered on day 0 and 2 postimmunization. The mice were monitored for clinical signs of EAE and scored. i-iii Mean values for all the mice in a group regardless of the presence or absence of clinical signs of EAE obtained from three individual experiments are indicated. iv Mean values for only the mice that showed clinical EAE in all of the above three experiments are shown as a pool. (b) Induction of EAE by adoptive transfer. Short-term $\mathrm{T}$ cell lines were derived from mice immunized with PLP 139-151 or ACA 83-95 and viable lymphoblasts were injected into naive SJL mice intravenously, and the mice were monitored for signs of EAE and scored. Mean values for a group of mice are shown. 
Table 2

Clinical and histologic EAE in SJL mice induced with PLP 139-151 and ACA 83-95. ${ }^{\text {a }}$

Clinical disease

Mean day of onset ${ }^{c}$

Mean maximum score

No. of inflammatory foci

Incidence (\%)

$11.18 \pm 0.44 \quad 3.39 \pm 0.28$

PLP 139-151

$18 / 18(100)$

$11.70 \pm 1.21$

$2.20 \pm 0.29$

0.011418

Meninges

Parenchyma

Total

ACA 83-95

$10 / 22(45)$

$45.44 \pm 8.88$

$5.59 \pm 1.57$

$40.00 \pm 9.63$

$85.44 \pm 17.84$

$p$ values

$6.83 \pm 1.01$

$4.50 \pm 0.34$

2.3416E-23

$3.77 \pm 0.9$

$9.36 \pm 2.15$

Adoptive transfer

PLP 139-151

$6 / 6$ (100)

$7.83 \pm 1.33$

$4.67 \pm 0.33$

$182.17 \pm 19.39$

$136.83 \pm 13.65$

$122.17 \pm 27.45$

$100.67 \pm 16.94$

$304.33 \pm 46.56$

Scoring scale: 0 , healthy; 1 , limp tail or hind limb weakness but not both; 2 , limp tail and hind limb weakness; 3 , partial paralysis of hind limbs; 4 , complete paralysis of hind limbs; and 5, moribund or dead.

${ }^{a}$ Numbers are mean \pm SEM.

'In this group, tissues from one mouse were retained for another aspect of the study.

'Represents only mice that showed clinical disease.

ing was specific since there was negligible binding to control tetramers (TMEV 70-86). Importantly, tetramer analysis also revealed positive staining for unimmunized cross-reacting peptides in cultures stimulated with the immunizing peptides. That is, cultures derived from mice immunized with PLP 139-151 showed positive staining for ACA 83-95 tetramers and vice versa (Figure 5b). However their

\section{(i) Active immunization}

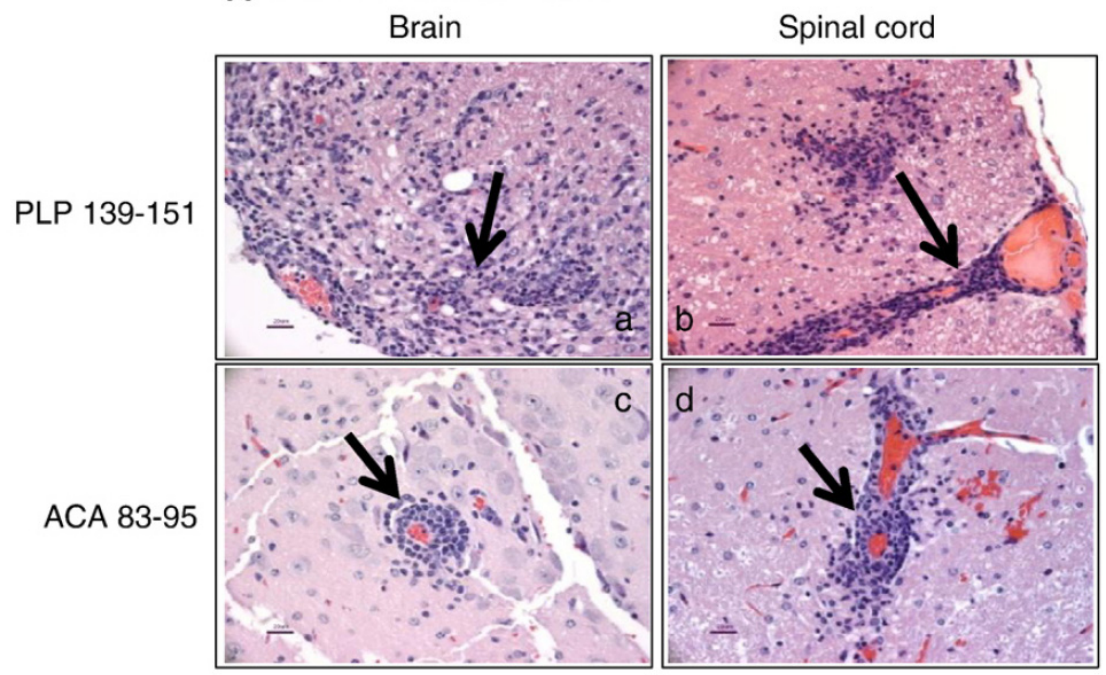

(ii) Adoptive transfer

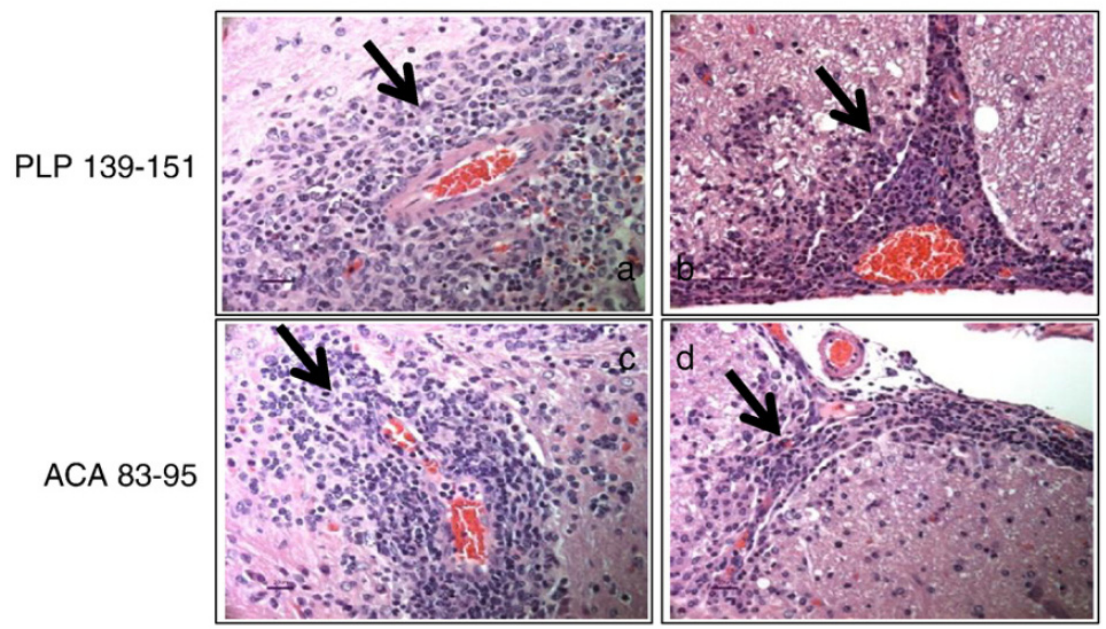

Figure 3. Histological evaluation of EAE induced with PLP 139-151 and ACA 83-95. (i) EAE induced by active immunization. Brain (a) and spinal cord (b) of PLP 139-151-immunized mouse with extensive perivascular lymphocytic infiltration (arrows) as compared with mild lymphocytic perivascular cuffing (arrow) in brain (c) and spinal cord (d) of ACA 83-95-immunized mouse. (ii) Similar lymphocytic mixed perivascular cuffing (arrows) in the brain and spinal cord of mice that received PLP 139-151 ( $a$ and b) and ACA 83-95 ( $c$ and d) -specific T cells. Original magnification, $\times 400(\mathrm{bar}=20 \mu \mathrm{m})$. 
frequency is less than that observed with the immunizing peptides. We further verified the $\mathrm{Ag}$ specificity of cross-reactive $\mathrm{T}$ cells for PLP 139-151 and ACA 83-95 in the mice immunized with the corresponding peptides by double tetramer staining using the tetramers conjugated with two different dyes namely APC and PE. Figure $5 c$ shows a fraction of CD4 T cells generated in response to PLP 139-151 or ACA 83-95 stained with both PLP 139-151 and ACA 83-95 tetramers, proving further that their T cells cross-react with each peptide. Notably, while the majority of ACA 83-95-reactive T cells that are generated in response to PLP 139151 immunization also stained with PLP 139-151 tetramers, only about a third of the ACA 83-95-reactive T cells generated in response to ACA 83-95-immunization stained with PLP 139-151 tetramers (Figure 5c).

3.5. Both ACA 83-95 and PLP 139-151 induce similar cytokine profiles

Cytokine secretion was tested by intracellular staining to enumerate the frequency of cytokine-secreting cells and in culture supernatants by ELISA (Figure 6). The cytokine panel consisted of $\mathrm{T}$ helper (Th) 1 (IL-2 and IFN-.), Th2 (IL-4 and IL-10), Th17 (IL-17A, IL-17F and IL22), and TNF-a. By analyzing the frequency of cytokine-secreting cells in the live CD4 T cell population, it was evident that the cells capable of secreting all of the cytokines tested were present in cultures stimulated with PLP 139-151 and ACA 83-95 and that the difference between the two groups was not significant (Figure 6a). While both PLP 139-151 and ACA 83-95 induced predominantly Th1 and Th17 cytokine production, the frequency of the combined cytokinesecreting CD4 T cells with respect to Th1, but not Th17 subset tended to be lower in ACA 83-95-than in PLP 139-151-stimulated

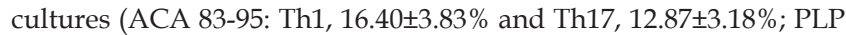
139151: Th1, $22.27 \pm 5.38 \%$ and Th17, $13.66 \pm 1.89 \%)(n=4)$. Similar trends were noted when cytokine secretion was evaluated by ELISA (Figure 6b) with no significant differences between the mice immunized with PLP 139-151 and ACA 83-95.

\section{Discussion}

We have identified a peptide mimic from the protozoan parasite, A. castellanii that induces clinical signs of EAE by generating crossreactive T cell responses with CNS myelin PLP 139-151. Microbial peptides that mimic myelin antigens capable of inducing CNS autoimmunity have been described and many of these are derived from pathogens that can cause CNS diseases in humans implying that they may have a role in the pathogenesis of autoimmune encephalomyelitis. It is also true that the microbial mimics generate cross-reacting $\mathrm{T}$ cell responses, but are unable to induce EAE as in the case of Haemophilus influenzae (HAE), 574-586 bearing homologous residues with PLP 139-151 (Carrizosa et al., 1998). Furthermore, microbial mimic

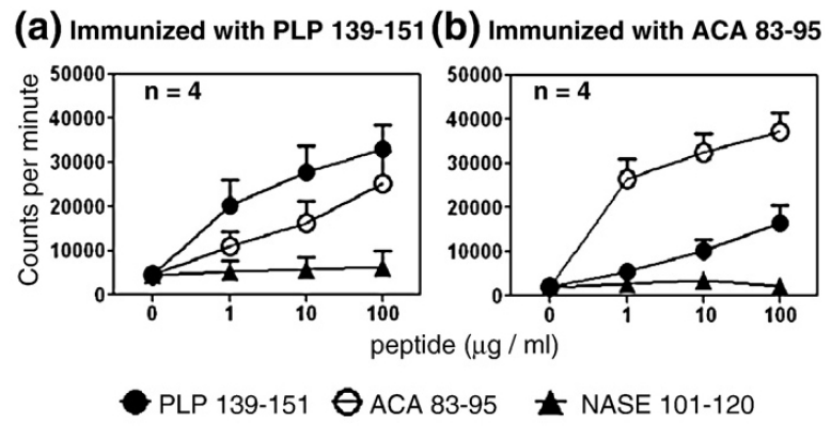

Figure 4. PLP 139-151 and ACA 83-95 induce cross-reactive T cell responses. Groups of SJL mice were immunized with PLP 139-151 or ACA 83-95 and after 10 days, mice were euthanized and lymph nodes were harvested. Lymph node cells were stimulated with PLP 139-151, ACA 83-95 and NASE 101-120 (control) for two days. Cells were then pulsed with tritiated ${ }^{3}[\mathrm{H}]$ thymidine, the incorporation of which was measured as cpm $16 \mathrm{~h}$ later. Mean \pm SEM values for a group of mice are shown $(n=4)$. sequences can act as natural altered peptide ligands and prevent autoimmune responses. For example, a peptide from human papilloma virus and Bacillus subtilis generate $\mathrm{T}$ cell responses that cross-react with MBP 87-99, but they prevent the induction of EAE (Ruiz et al., 1999). These observations suggest that microbial exposure does not necessarily mean that detrimental organ-specific autoimmune responses ensue. Nonetheless, identifying peptides from microbes that are known to cause CNS illness provides opportunities to investigate their potential involvement in the initiation of autoimmune diseases. We chose ACA 83-95, BCE 204-216 and MTB 64-76 to test their disease-inducing potential and of these, only ACA 83-95 induced the clinical signs of EAE by active immunization. It has been previously reported that HAE 574-586 was found to generate T cell responses that cross-react with PLP 139-151, but was unable to induce EAE by active immunization except in mice primed with suboptimal doses of PLP 139-151 (Carrizosa et al., 1998) or in mice in which a preexisting population of HAE 574-586-specific T cells was created by viral delivery (Croxford et al., 2005). ACA $83-95$ differs from HAE 574-586 in that it possesses histidine at position 147 as an additional residue. The fact that ACA peptide, but not HAE peptide, induces EAE suggests that the presence of both primary (tryptophan, position 144) and secondary contact residues (leucine, position 141; and histidine, position 147) are essential to induce encephalitogenic $\mathrm{T}$ cell responses (Figure 1 ). This may be why HAE $574-586$ by itself failed to induce EAE. In the case of two other peptides, namely BCE 204-216 and MTB 6476, the secondary contact residue was present, but failed to induce EAE or generated cross-reactive T cell responses, and they were poorly immunogenic (data not shown). It may be that they bind weakly to MHC and/or TCR or that some of the non-critical TCR and MHC residues in the non-immunogenic peptides may be essential for maintaining the conformation of the epitope for efficient TCR and MHC recognition as previously speculated (Croxford et al., 2005).

Comparison of the incidence and EAE severity induced by PLP 139151 with that of ACA 83-95 revealed that ACA 83-95 induced clinical and/or histological EAE in only $72.7 \%$ of animals and the disease severity was mild (Figure 2 and Table 2). Lack of $100 \%$ incidence and the less severe nature of EAE induced with ACA 83-95 suggest three possibilities: (a) ACA 83-95-induced cross-reactive T cell responses with PLP 139-151 are less encephalitogenic than those that can be obtained from immunization with native PLP 139-151 peptide. (b) The generation of the precursor frequency of cross-reactive T cells induced with ACA 8395 immunization might be low and (c) the functional phenotype of ACA 83-95-induced T cell population might be different with respect to the production of inflammatory cytokines. We tested whether the $\mathrm{T}$ cells generated in response to ACA 83-95 can induce EAE in adoptive transfer experiments. Both the T cells that react with PLP 139-151 and ACA 83-95 induced the clinical signs of EAE in a similar manner clinically (Figure $2 b$ ) and histologically (Table 2 and Figure 3ii) indicating that ACA 83-95 can generate potent autoaggressive T cells and induce EAE. Reports indicate that the naturally occurring Acanthamoeba granulomatous encephalitis (AGE) induced with ACA accompanies infiltrations consisting of MNC, plasma cells, and multinucleated giant cells (Marciano-Cabral and Cabral, 2003). In our studies with ACA 83-95, although MNC were present, the dominance of plasma cells and giant cells was absent. This lack of similarity is not surprising, because the pathology that occurs in AGE is due to the presence of infectious organism in the brain which may involve the mediation of multiple factors and different cell types.

Since both PLP 139-151- and ACA 83-95-reactive T cells induced EAE with a similar severity; we asked if the differences in disease severity induced by active immunization are due to variations in the generation of precursor frequency of Ag-specific T cells. Figure 4 shows that both PLP 139-151 and ACA 83-95 induce T cell responses for both immunizing and unimmunizing peptides in a criss-cross manner. Importantly, the reactivity to PLP 139-151 in ACA 83-95-immunized mice was marginally low, which raised the question of whether the generation of the precursor frequency of the disease-in- 
(a) PLP 139-151 tetramer staining

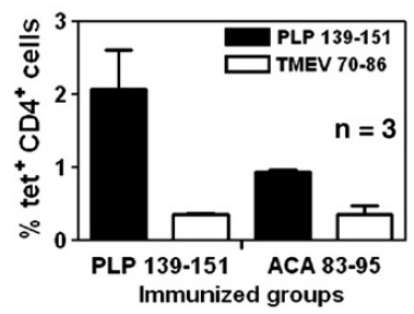

(c) Double tetramer staining Immunized groups

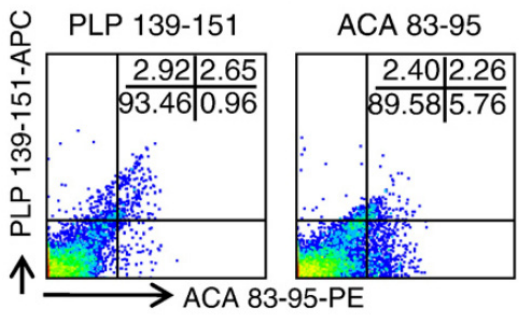

(b) Cross reactivity Immunized groups

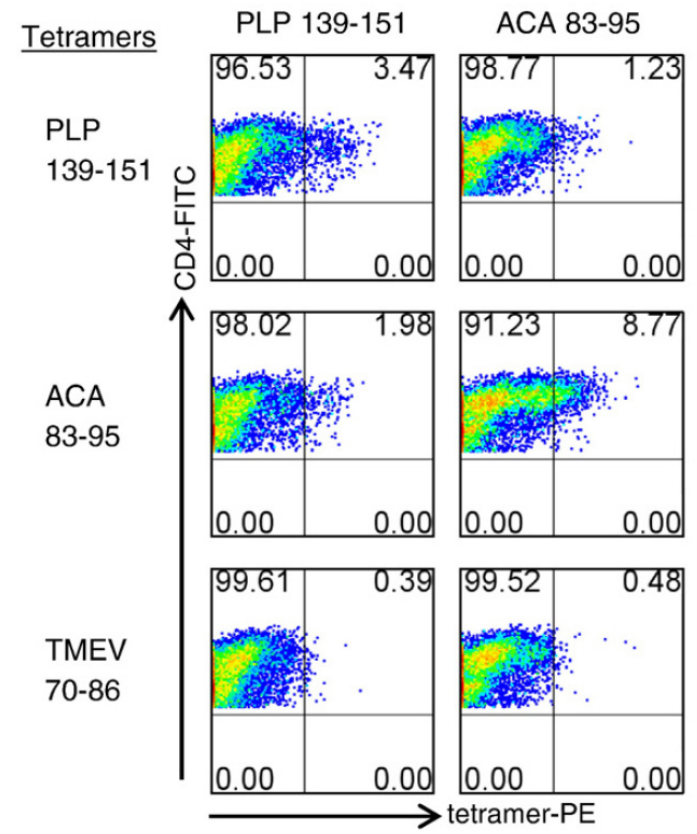

Figure 5. Cross-reactive T cell responses induced by PLP 139-151 and ACA 83-95 are Ag-specific. (a) IAs/PLP 139-151 tetramers detect PLP 139-151-reactive T cells in mice immunized with ACA 83-95. LNC obtained from SJL mice immunized with PLP 139-151 or ACA 83-95 were restimulated with the corresponding peptides and tetramer staining was performed flow cytometrically by using PE-conjugated PLP 139-151 and TMEV 70-86 tetramers, anti-CD4 and 7-AAD as described in Materials and methods section. Percentages of tet+CD4+ T cells were enumerated in live (7-AAD-) populations. Mean \pm SEM values are shown $(n=3$ per group). (b) Cross-reactive T cells induced by PLP 139-151 and ACA 83-95 are Ag-specific. IAs tetramers involving PLP 139-151, ACA 83-95 and TMEV 70-86-PE were used to stain LNC stimulated with PLP 139-151 or ACA 83-95 and analyzed by flow cytometry. Tet+ cells in CD4 subset are shown. (c) Detection of cross-reactive T cell responses by double tetramer staining. APC-conjugated PLP 139-151 and PE-conjugated ACA 83-95 tetramers were used to stain LNC stimulated with PLP 139-151 or ACA 83-95 and the tet+ cells are shown in CD4 subset. In all of the staining reactions, TMEV 70-86 tetramers were included as negative controls and 7-AAD as a cell death marker.

ducing PLP 139-151-specific T cells is low in ACA 83-95-immunized mice. We confirmed these differences in the expansion of Ag-specific $\mathrm{T}$ cells by tetramer staining (Figure 5). The lesser magnitude in the generation of PLP 139-151-specific T cells in ACA 83-95-immunized (a) Intracellular staining

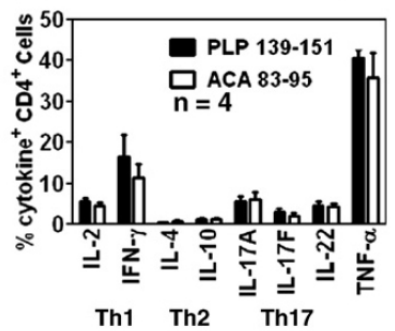

\section{(b) Cytokine ELISA}

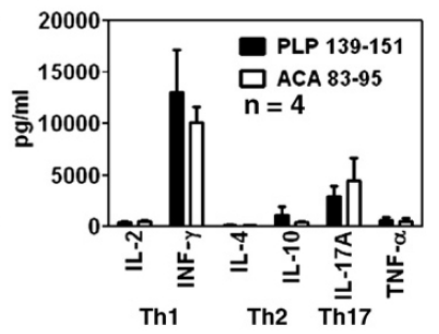

Figure 6. PLP 139-151 and ACA 83-95 induce identical cytokine patterns. (a) Cytokine detection by intracellular staining. Lymph node cells derived from SJL mice immunized with PLP 139-151 or ACA 83-95 were stimulated with the corresponding peptides for two days and the cultures were maintained in IL-2 medium for additional two days. Viable lymphoblasts were harvested and stimulated with PMA and ionomycin for $4.5 \mathrm{~h}$ in the presence of monensin and stained with anti-CD4 and 7-AAD. After fixation and permeabilization, the cells were incubated with cytokine antibodies and the frequency of cytokine $^{+}$cells was enumerated by flow cytometry in the live (7-AAD) $\mathrm{CD}^{+}$ T cells. Percentages of cytokine-secreting CD4 T cells for a group of mice are shown $(n=4)$. (b) Cytokine detection by ELISA. Supernatants harvested on day two poststimulation with peptides from the above cultures were analyzed for cytokines by ELISA. Each bar represents mean \pm values for a group of mice $(n=4)$. mice could explain the lack of $100 \%$ disease incidence in the mice immunized with ACA 83-95. This is likely because the mice that received PLP 139151- and ACA 83-95-specific T cells developed EAE with a similar disease phenotype, both clinically and histologically (Figures $2 \mathrm{~b}$ and $3 \mathrm{ii}$ and Table 2).

To determine whether differences in the severity of EAE induced with ACA 83-95 and PLP 139-151 might be due to variations in the production of cytokines, we evaluated cytokine secretion in LNC obtained from PLP 139-151-and ACA 83-95-immunized mice, but it was not the case (Figure 6). It has been recently suggested that the production of Th17 cytokines marks the generation of potent autoaggressive T cell populations (McGeachy and Cua, 2008) and we did not see such a skewed response to either of the two peptides. It is likely that the T cells capable of producing both Th1 and Th17 cytokines may have contributed to the disease phenotypes. Although both Th1 and Th17 cells could contribute to EAE pathogenicity, it appears that the ratio between the two subsets of T cells determines the severity of EAE in that predominance of Th17 over Th1 cells exacerbates inflammation and infiltration into the CNS (Bettelli et al., 2007; Stromnes et al., 2008). Furthermore, it has been proposed that Th1 cells enter non-inflamed CNS tissues prior to Th17 cells and initiate inflammation and that they facilitate the entry of Th17 cells (O'Connor et al., 2008). We have observed that ACA 83-95 induced a relatively lesser proportion of Th1 cells when compared with PLP 139151 (16.40 vs. $22.27 \%)$ and this difference in the generation of Th1 cells might have contributed to the less severe nature of EAE induced with ACA 83-95.

Genetic susceptibility and environmental factors predispose to the development of autoimmune responses. It is possible that exposure to pathogens that bear homologous sequences with self-antigens 
could lead to the generation of cross-reactive $\mathrm{T}$ cells both in periphery and locally within the CNS. Myelin antigens such as MBP and PLP can be expressed in thymus and peripheral lymphoid tissues and aberrant expression of PLP transcripts importantly DM20, an isoform of PLP lacking PLP 139-151 motif contribute to the endogenous generation of PLP 139-151 reactive T cells by escaping central tolerance (Anderson et al., 2000; Klein et al., 2000; Voskuhl, 1998). Furthermore, healthy humans including MS patients react to MBP or PLP suggestive of the existence of endogenous myelin reactive $\mathrm{T}$ cell repertoires and the resident dendritic cells and microglia can present antigens locally within CNS (Bailey et al., 2007; Pette et al., 1990; Wucherpfennig and Strominger, 1995). Therefore, it is likely that during the course of natural infection with ACA, the resident Ag-presenting cells can present a peptide encompassing 83-95, stimulate the expansion of PLP 139-151-reactive CD4 T cells by molecular mimicry, and induce autoimmunity in the CNS.

In summary, we have provided evidence that ACA 83-95 derived from a protozoan parasite can induce CNS autoimmunity by molecular mimicry. ACA is a free-living organism present throughout the world habituating in water, soil, and dust, and it causes AGE, amoebic keratitis and cutaneous amoebiasis in infected humans (Claerhout and Kestelyn, 1999; Marciano-Cabral and Cabral, 2003). AGE is a chronic progressive disease of the CNS commonly seen in chronically ill, debilitated, or immunocompromised individuals such as AIDS patients (Marciano-Cabral and Cabral, 2003). The amoebae activate phosphatidylinositol 3-kinase to induce apoptosis of brain microvascular endothelial cells and successfully traverse the blood-brain barrier to induce AGE (Sissons et al., 2005). IL-1B has been implicated in the formation of granuloma in the CNS tissues, but it is unclear whether the hemorrhagic necrosis caused by Acanthamoeba infections results from destruction of brain tissue or inflammatory cytokines (Marciano-Cabral et al., 2004). Presently, it is not known if natural infection with ACA trophozoites can lead to the generation of PLP-reactive T cells or T cell clones specific to other myelin antigens, which could be released as a consequence of epitope spreading (Miller et al., 1997). Molecular mimicry has been proposed as one of the phenomena for the occurrence of a number of autoimmune diseases (Ercolini and Miller, 2005). Although not known presently, our data provide a rationale to test whether there is any possible association of ACA infection with the occurrence of autoimmune encephalomyelitis. It may be possible that in genetically susceptible individuals who are immunocompromised, exposure to ACA can trigger CNS autoimmunity by molecular mimicry.

\section{Acknowledgements}

We thank Drs. Thomas Petro and Rana Zabad for critical reading of this manuscript and Jean-Jack Riethoven for statistical analysis.

\section{References}

Anderson, A.C., Nicholson, L.B., Legge, K.L., Turchin, V., Zaghouani, H., Kuchroo, V.K., 2000. High frequency of autoreactive myelin proteolipid protein-specific T cells in the periphery of naive mice: mechanisms of selection of the self-reactive repertoire. J. Exp. Med. 191: 761-770.

Bailey, S.L., Schreiner, B., McMahon, E.J., Miller, S.D., 2007. CNS myeloid DCs presenting endogenous myelin peptides 'preferentially' polarize CD4+ T(H)-17 cells in relapsing EAE. Nat. Immunol. 8: 172-180.

Bettelli, E., Oukka, M., Kuchroo, V.K., 2007. T(H)-17 cells in the circle of immunity and autoimmunity. Nat. Immunol. 8: 345-350.

Brocke, S., Gaur, A., Piercy, C., Gautam, A., Gijbels, K., Fathman, C.G., Steinman, L., 1993. Induction of relapsing paralysis in experimental autoimmune encephalomyelitis by bacterial superantigen. Nature 365: 642-644.

Carrizosa, A.M., Nicholson, L.B., Farzan, M., Southwood, S., Sette, A., Sobel, R.A., Kuchroo, V.K., 1998. Expansion by self antigen is necessary for the induction of experimental autoimmune encephalomyelitis by $\mathrm{T}$ cells primed with a cross-reactive environmental antigen. J. Immunol. 161: 3,307-3,314.
Claerhout, I., Kestelyn, P., 1999. Acanthamoeba keratitis: a review. Bull. Soc. Belge Ophtalmol. 274, 71-82. Conant, S.B., Swanborg, R.H., 2003. MHC class II peptide flanking residues of exogenous antigens influence recognition by autoreactive T cells. Autoimmun. Rev. 2: 8-12.

Croxford, J.L., Anger, H.A., Miller, S.D., 2005. Viral delivery of an epitope from Haemophilus influenzae induces central nervous system autoimmune disease by molecular mimicry. J. Immunol. 174: 907-917.

Ercolini, A.M., Miller, S.D., 2005. Role of immunologic cross-reactivity in neurological diseases. Neurol. Res. 27: 726-733.

Fujinami, R.S., Oldstone, M.B., 1985. Amino acid homology between the encephalitogenic site of myelin basic protein and virus: mechanism for autoimmunity. Science 230: 1,043-1,045.

Fujinami, R.S., von Herrath, M.G., Christen, U., Whitton, J.L., 2006. Molecular mimicry, bystander activation, or viral persistence: infections and autoimmune disease. Clin. Microbiol. Rev. 19: 80-94.

Gautam, A.M., Liblau, R., Chelvanayagam, G., Steinman, L., Boston, T., 1998. A viral peptide with limited homology to a self peptide can induce clinical signs of experimental autoimmune encephalomyelitis. J. Immunol. 161: 60-64.

Kerlerode Rosbo, N., Milo, R., Lees, M.B., Burger, D., Bernard, C.C., Ben-Nun, A., 1993. Reactivity to myelin antigens in multiple sclerosis. Peripheral blood lymphocytes respond predominantly to myelin oligodendrocyte glycoprotein. J. Clin. Invest. 92: 2,6022,608 .

Klein, L., Klugmann, M., Nave, K.A., Tuohy, V.K., Kyewski, B., 2000. Shaping of the autoreactive T-cell repertoire by a splice variant of self protein expressed in thymic epithelial cells. Nat. Med. 6: 56-61.

Mao, Y.S., Lu, C.Z., Wang, X., Xiao, B.G., 2007. Inductionofexperimental autoimmune encephalomyelitis in Lewis rats by a viral peptide with limited homology to myelin basic protein. Exp. Neurol. 206: 231-239.

Marciano-Cabral, F., Cabral, G., 2003. Acanthamoeba spp. as agents of disease in humans. Clin. Microbiol. Rev. 16: 273-307.

Marciano-Cabral, F., Ludwick, C., Puffenbarger, R.A., Cabral, G.A., 2004. Differential stimulation of microglial pro-inflammatory cytokines by Acanthamoeba culbertsoni versus Acanthamoeba castellanii. J. Eukaryot. Microbiol. 51: 472-479.

McGeachy, M.J., Cua, D.J., 2008. Th17 cell differentiation: the long and winding road. Immunity 28: 445-453.

Mendel, I., Kerlero de Rosbo, N., Ben-Nun, A., 1995. A myelin oligodendrocyte glycoprotein peptide induces typical chronic experimental autoimmune encephalomyelitis in $\mathrm{H}-2 \mathrm{~b}$ mice: fine specificity and $\mathrm{T}$ cell receptor $\mathrm{V}$ beta expression of encephalitogenic $\mathrm{T}$ cells. Eur. J. Immunol. 25: 1,951-1,959.

Miller, S.D., Vanderlugt, C.L., Begolka, W.S., Pao, W., Yauch, R.L., Neville, K.L., Katz-Levy, Y., Carrizosa, A., Kim, B.S., 1997. Persistent infection with Theiler's virus leads to CNS autoimmunity via epitope spreading. Nat. Med. 3: 1,133-1,136.

Mokhtarian, F., Zhang, Z., Shi, Y., Gonzales, E., Sobel, R.A., 1999. Molecular mimicry between a viral peptide and a myelin oligodendrocyte glycoprotein peptide induces autoimmune demyelinating disease in mice. J. Neuroimmunol. 95: 43-54.

Nicholson, L.B., Greer, J.M., Sobel, R.A., Lees, M.B., Kuchroo, V.K., 1995. An altered peptide ligand mediates immune deviation and prevents autoimmune encephalomyelitis. Immunity 3: 397-405.

O'Connor, R.A., Prendergast, C.T., Sabatos, C.A., Lau, C.W., Leech, M.D., Wraith, D.C., Anderton, S.M., 2008. Cutting edge: Th1 cells facilitate the entry of Th17 cells to the central nervous system during experimental autoimmune encephalomyelitis. J. Immunol. 181: 3,750-3,754.

Pette, M., Fujita, K., Wilkinson, D., Altmann, D.M., Trowsdale, J., Giegerich, G., Hinkkanen, A., Epplen, J.T., Kappos, L., Wekerle, H., 1990. Myelin autoreactivity in multiple sclerosis: recognition of myelin basic protein in the context of HLA-DR2 products by T lymphocytes of multiple-sclerosis patients and healthy donors. Proc. Natl. Acad. Sci. U.S.A. 87: 7,968-7,972. 
Reddy, J., Bettelli, E., Nicholson, L., Waldner, H., Jang, M.H., Wucherpfennig, K.W., Kuchroo, V.K., 2003. Detection of autoreactive myelin proteolipid protein 139-151-specific T cells by using MHC II (IA') tetramers. J. Immunol. 170: 870-877.

Reddy, J.,Illés, Z., Zhang, X., Encinas, J., Pyrdol, J., Nicholson, L., Sobel, R.A., Wucherpfennig, K.W., Kuchroo, V.K., 2004. Myelin proteolipid protein-specific $\mathrm{CD} 4+\mathrm{CD} 25+$ regulatory cells mediate genetic resistance to experimental autoimmune encephalomyelitis. Proc. Natl. Acad. Sci. U.S.A. 101: 15,434-15,439.

Ruiz, P.J., Garren, H., Hirschberg, D.L., Langer-Gould, A.M., Levite, M., Karpuj, M.V., Southwood, S., Sette, A., Conlon, P., Steinman, L., 1999. Microbial epitopes act as altered peptide ligands to prevent experimental autoimmune encephalomyelitis. J. Exp. Med. 189: 1,275-1,284.

Sissons, J., Kim, K.S., Stins, M., Jayasekera, S., Alsam, S., Khan, N.A., 2005. Acanthamoeba castellanii induces host cell death via a phosphatidylinositol 3-kinase-dependent mechanism. Infect. Immun. 73: 2,704-2,708

Sobel, R.A., Tuohy, V.K., Lu, Z.J., Laursen, R.A., Lees, M.B., 1990. Acute experimental allergic encephalomyelitis in SJL/J mice induced by a synthetic peptide of myelin proteolipid protein. J. Neuropathol. Exp. Neurol. 49: 468-479.
Sospedra, M., Martin, R., 2005. Immunology of multiple sclerosis. Annu. Rev. Immunol. 23, 683-747.

Stromnes, I.M., Cerretti, L.M., Liggitt, D., Harris, R.A., Goverman, J.M., 2008. Differential regulation of central nervous system autoimmunity by $\mathrm{T}(\mathrm{H}) 1$ and $\mathrm{T}(\mathrm{H}) 17$ cells. Nat. Med. 14, 337-342.

Tuohy, V.K., Lu, Z., Sobel, R.A., Laursen, R.A., Lees, M.B., 1989. Identification of an encephalitogenic determinant of myelin proteolipid protein for SJL mice. J. Immunol. 142, 1523-1527.

Voskuhl, R.R., 1998. Myelin protein expression in lymphoid tissues: implications for peripheral tolerance. Immunol. Rev. 164, 81-92.

Whitham, R.H., Bourdette, D.N., Hashim, G.A., Herndon, R.M., Ilg, R.C., Vandenbark, A.A., Offner, H., 1991. Lymphocytes from SJL/J mice immunized with spinal cord respond selectively to a peptide of proteolipid protein and transfer relapsing demyelinating experimental autoimmune encephalomyelitis. J. Immunol. 146, 101-107.

Wucherpfennig, K.W., Strominger, J.L., 1995. Molecular mimicry in $\mathrm{T}$ cell-mediated autoimmunity: viral peptides activate human $\mathrm{T}$ cell clones specific for myelin basic protein. Cell 80, 695-705.

Zamvil, S.S., Mitchell, D.J., Moore, A.C., Kitamura, K., Steinman, L., Rothbard, J.B., 1986. T-cell epitope of the autoantigen myelin basic protein that induces encephalomyelitis. Nature 324, 258-260. 